\title{
Wishart Matris'lerinde En Büyük Özdeğer Olasılık Dağılım Fonksiyonlarının Karşılaştırılması ${ }^{*}$
}

\author{
Hüsamettin Çelik ${ }^{1 * *}$, Nurhan Karaboğa ${ }^{2}$ \\ ${ }^{1}$ Erzincan Binali Yıldırım Üniversitesi, Tercan Meslek Yüksekokulu, Bilgisayar Teknolojileri Bölümü, Erzincan, Türkiye (ORCID: 0000-0002-7662-0674) \\ ${ }^{2}$ Erciyes Üniversitesi, Mühendislik Fakültesi, Elektrik-Elektronik Mühendisliği Bölümü, Kayseri, Türkiye (ORCID: 0000- 0002-4550-5251)
}

((Konferans Tarihi: 5-7 Mart 2020)

(DOI: 10.31590/ejosat.araconf51)

ATIF/REFERENCE: Çelik, H. \& Karaboğa N. (2020). Wishart Matris'lerinde En Büyük Özdeğer Olasıllk Dağılım Fonksiyonlarının Karşılaştırılması. Avrupa Bilim ve Teknoloji Dergisi, (Özel Sayı), 393-398.

$\ddot{O} \mathbf{z}$

Wishart matrisleri ve bu matrislerin özdeğer dağılımları telekomünikasyon, algılama teorisi ve çok değişkenli veri analizlerinde oldukça yaygın olarak kullanılmaktadır. Fakat bu dağılımlar önceden tanımlanmış herhangi fonksiyona benzemediğinden, kapalı bir fonksiyonla ifade edilmesi çok kolay olmamaktadır. Literatürde özellikle Wishart matrislerinin en büyük özdeğerinin olasılık dağılımı için yapılan çalışmalar mevcuttur. Bu çalışmalarda Wishart matrislerinin en büyük özdeğerine eklenen parametrelerle 1. dereceden Tracy-Widom dağılımına benzetilmektedir. Fakat dağılımın benzerlik ölçüsü eklenen parametrelere göre değişmektedir. Yapılan bu çalışma, Ma, Jhonstone, Deo tarafından önerilen en büyük özdeğer dağılım fonksiyonlarının performanslarını karşılaştırmayı amaçlamıştır. Benzetim çalışmaları farklı boyuttaki matrisler için uygulanmış ve sonuçlar karşılaştırılmışıı. Yapplan benzetim çalışmalarına göre, Deo tarafından sunulan en büyük özdeğer dağılım fonksiyonunun, 1. dereceden Tracy-Widom olasılık dağılımına en fazla benzediği görülmüştür.

\section{Performance Analysis of Blind Source Algorithms in the Separation of Sound Signals}

\begin{abstract}
Wishart matrices and eigenvalue distributions of these matrices are widely used in telecommunication, perception theory and multivariate data analysis. However, since these distributions do not resemble any predefined functions, it is not easy to express them with a closed function. There are studies in the literature, especially for the probability distribution of the largest eigenvalue of Wishart matrices. In these studies, some parameters are added to the largest eigenvalue of Wishart matrices and they are likened to Tracy-Widom distribution of order 1 . However, the similarity of the distribution varies according to the added parameters. This study aimed to compare the performances of the largest eigenvalue distribution functions proposed by Ma, Jhonstone, Deo. Simulation studies were applied for matrices with different dimension and the results were compared. According to the simulation studies, the largest eigenvalue distribution function presented by Deo was found to be most similar to the first order Tracy-Widom probability distribution.
\end{abstract}

\footnotetext{
* Bu makale International Conference on Access to Recent Advances in Engineering and Digitalization (ARACONF 2020) de sunulmuştur.

** Sorumlu Yazar: Erzincan Binali Yıldırım Üniversitesi, Tercan Meslek Yüksekokulu, Bilgisayar Teknolojileri Bölümü, Erzincan, Türkiye, ORCID: 0000-0002-7662-0674, hcelik@,erzincan.edu.tr
} 
Keywords: Covariance matrix, Maximum eigenvalue, Probability distribution function, Tracy-Widom probability distribution, Wishart matrix.

\section{Giriș}

Rassal matrislerin özdeğer dağılımları ve bu dağılımların kapalı fonksiyonları, haberleşme sistemleri, haberleşme kanallarının kapasite hesapları ve birçok hipotez testleri için kullanılmaktadır (Ciflikli \& Ilgin, 2017; 2020). Bunların yanı sıra Wishart ve Gauss dağılımlı rassal matrislerinin en büyük özdeğerlerinin dağılımı, temel bileşen analizi de dahil olmak üzere çok değişkenli veri analizi alanında önemli rol oynaktadır (Edelman, 2005; Johnstone, 2001). Özellikle Bilişsel Radyo uygulamaları ve spektrum algılama yöntemlerinde özdeğer dağılım fonksiyonlarına oldukça fazla ihtiyaç duyulmaktadır (Chen \& Zhang, 2018; Mohammadi, Javadi, Ciuonzo, Persico, \& Pescapè, 351). Bunlara ek olarak haberleşme sistemlerinin kanal kapasite hesapları da yine özdeğer dağılım fonksiyonlarına dayanmaktadır. Bu nedenle karmaşık veya gerçek sonlu matrisler için en büyük öz değerin olasılık dağılım fonksiyonları kapalı olarak bazı çalışmalarda incelenmiştir (Ciflikli \& Ilgin, 2017; Ma, 2012). Bu çalışmalardan birinde en büyük özdeğere çeşitli parametreler eklenerek, dağılımı 1. dereceden Tracy-Widom ( $\left.\mathrm{TW}_{1}\right)$ olasılık dağılımına benzetilmeye çalışılmıştır

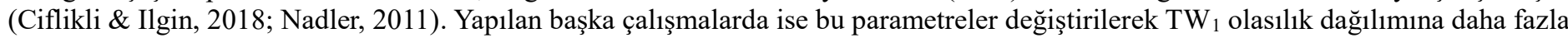
yakınsadı̆̆ı gözlemlenmiştir. Ayrıca en büyük özdeğerin olasılık dağılımını belirli kapalı fonksiyonlara benzetmek amacı ile farklı yöntemlerde uygulanmıştır. Yapılan bu çalışmaların hepsinin ortak amacı en küçük matris boyutlarında, özdeğer dağılımını belirli fonksiyonlara benzetmektir (Johansson, 2007).

Bu çalışmanın amacı ise literatürde bulunan en büyük özdeğer dağılımları için kullanılan bazı fonksiyonları karşılaştırmaktır. Bu bağlamda Wishart matrislerinin en büyük özdeğer dağılımını, TW $\mathrm{TW}_{1}$ 'e benzetmek için çalışmalar yapılmış ve bunlar değerlendirilmiştir. Yapılan çalışmada farklı satır ve sütun sayısındaki Wishart matrisleri için farklı dağılım fonksiyonlarının analizi ve benzetim sonuçları verilmektedir.

Yapılan bu çalışmanın organizasyonu şu şekildedir: 2. Bölümde Wishart matrisleri ve bu matrislerin özdeğer dağılımları ile ilgili bilgiler verilmektedir. Benzetim çalışmalarında kullanılacak olan farklı olasılık dağılımlarının teorik ifadeleri de bu bölümde açıklanmaktadır. Yapılan çalışmanın benzetim sonuçları ve tartışma 3. Bölümde verilmektedir. Son olarak, 4. Bölüm’de ise sonuçlara yer verilmektedir.

Bu çalışmada küçük normal karakterler $(x)$ vektörleri, büyük koyu karakterler $(\boldsymbol{X})$ matrisleri temsil etmektedir. Ayrıca $\boldsymbol{X}^{\prime}$ ifadesi devrik (conjugate, transpoz) matrisi belirtmektedir.

\section{Materyal ve Metot}

\subsection{Wishart Matrisleri İçin En Büyük Özdeğer Dağılımı}

Örnek kovaryans matrisi özdeğerleri ve bu özdeğerlerin olasılık dağılım fonksiyonları çok değişkenli istatistiğin temel konularından biri olmakla beraber son yıllarda üzerinde fazlaca çalışılan konular arasındadır. $\boldsymbol{X}$, nxp boyutunda bağımsız aynı dağılımlı bir matris olmak üzere herhangi bir $\boldsymbol{W}$ matrisi $\boldsymbol{W}=\boldsymbol{X} \boldsymbol{X}^{\prime}$ çarpımları şeklinde yazılabiliyorsa $\boldsymbol{W}$ matrisi Wishart matrisi olarak adlandırılır ve bu matris Wishart dağılımına sahiptir. Burada $\boldsymbol{X}$ matrisi ( $x x p$ ) boyutunda karmaşık (complex) veya gerçek (real) olabilir ve $\boldsymbol{X}^{\prime}$ onun eşlenik devriğini (konjuge transpozunu) belirtir. $\mu_{x}, X$ matrisinin ortalamasını, $\boldsymbol{\Sigma}$ kovaryans matrisini göstermek üzere $\mu=0$ koşulu ile (nxn) boyutlarındaki kovaryans matrisi ise $\boldsymbol{\Sigma}=\boldsymbol{W}=\boldsymbol{X} \boldsymbol{X}^{\prime}$ olur.

Birçok hipotez testinde kovaryans matrisi, $\lambda_{1}>\lambda_{2}>\cdots>\lambda_{p}$ durumu için özdeğerlerinden faydalanılarak, küresellik testleri, gürültülü süreçlerde işaret algılama testleri gibi karar çıkarımları yapılmaktadır. $\sum=\sigma^{2} \boldsymbol{I}_{p}$ olması durumunu göz önünde bulundurarak bu varsayım altında $(n, p \rightarrow \infty)$ iken $\mathrm{e} \equiv p / n \epsilon(0, \infty)$ ve dağılımın varyansı $\sigma$, dağılımın merkezini gösteren $\mu$ olup aşağıdaki gibi tanımlanmaktadır.

$$
L_{j} \equiv\left(\dot{\lambda}_{1} / \sigma^{2}-\tilde{\mu}_{n, p}\right) / \tilde{\sigma}_{n, p} \stackrel{D}{\rightarrow} T W_{1}
$$

Bu eşitlik 1. dereceden Tracy-Widom dağılımına yakınsar ki bu dağılım rassal hermit matrisinin en büyük özdeğerinin olasılık dağılım fonksiyonunu belirtir (Johnstone, 2001).

Burada

$$
\begin{aligned}
& \tilde{\mu}_{n, p}=n^{-1}(\sqrt{n-1}+\sqrt{p})^{2} \\
& \tilde{\sigma}_{n, p}=\left(\frac{\widetilde{\mu}_{n, p}}{n}\right)^{\frac{1}{2}}\left(\frac{1}{\sqrt{n-1}}+\frac{1}{\sqrt{p}}\right)^{\frac{1}{3}}
\end{aligned}
$$

tanımlanmış olup 1. dereceden Tracy-Widom dağılımını belirtir.

Ma yaptığı çalışmalar ile merkez $\tilde{\mu}_{n, p}$ ve skala $\tilde{\sigma}_{n, p}$ katsayılarını,

$$
\mu_{n, p}=n^{-1}(\sqrt{n-1 / 2}+\sqrt{p-1 / 2})^{2}
$$




$$
\sigma_{n, p}=\left(\mu_{n, p} / n\right)^{1 / 2}\left(\frac{1}{\sqrt{n-1 / 2}}+\frac{1}{\sqrt{p-1 / 2}}\right)^{1 / 3}
$$

şeklinde değiştirmiş ve bu değişimle birlikte Eşitlik (6) ile verilen matematiksel ifade de sonlu örnek uzunlukları TW 1 dağılımına daha çok yakınsadığını ispatlamıştır (Ma, 2012).

$$
L_{m a} \equiv\left(\dot{\lambda}_{1} / \sigma^{2}-\mu_{n, p}\right) / \sigma_{n, p} \stackrel{D}{\rightarrow} T W_{1}
$$

$\mathrm{Bu}$ çalışmanın yanı sıra literatürde kovaryans matrisi özdeğer dağılımıyla ilgi oldukça fazla çalışma bulunmaktadır. Örneğin Nadler kuyruk olasılıklar için daha iyi bir yaklaşım elde edilebileceğini simülasyonlar yardımıyla göstermiştir (Nadler, 2011). Bu yaklaşım önemli bir gelişme sağlamakla beraber, hesaplama maliyeti dezavantajı ve uygun bir dağılım fonksiyonu olmadığı nedeniyle fazla kullanılmamıştır. Dahası, ikinci türev de negatif değerler alabileceğinden, fonksiyon negatif değerler üretebilir. Literatürde Wishart matrislerinin en büyük özdeğerleri ile ilgili yapılmış farklı çalışmalar da bulunmaktadır (Johnstone, 2001; Deo, 2016). Ancak, bu ifadeler hesaplama açısından daha maliyetlidir ve sadece karmaşık durum için geçerlidir. Nadler tarafından yapılan çalışma hesaplama açısından çok basit olmasıyla birlikte hem gerçek hem de karmaşık işaretlere uygulanabilir (Nadler, 2011). Belirli bir Wishart matrisinin varyans değerinin tahmini, oldukça önemlidir. Eğer Wishart matrisinin varyansı $\left(\sigma^{2}\right)$ bilinmiyorsa tahmini bir $\hat{\lambda}_{1}$ değeri kullanılarak geliştirilebilir. Bununla birlikte $\sigma^{2}$ tahmini için en basit ve kullanışlı kestirici $W$ matrisinin ortalama izidir (Ciflikli \& Ilgin, 2018; Deo, 2016).

$$
\boldsymbol{\sigma}_{\mathbf{0}}^{2} \equiv p^{-1} \operatorname{trace}(\boldsymbol{W})=p^{-1} \sum_{i=1}^{p} \hat{\lambda}_{1}
$$

Geliştirilmiş özdeğer yaklaşımı için 1. dereceden Tracy-Widom dağılımına bakmak gerekli ve yeterlidir. Bu dağılımda $a \equiv$ $E\left(W_{1}\right)=1.267941$ ve $b \equiv \sqrt{\operatorname{var}\left(W_{1}\right)}=1.267941$ olmak şartıyla $W_{1} \equiv W_{1}-a / b$ olmaktadır ve sabit parametreler,

$$
\begin{aligned}
& \mu_{n, p, 0}=\mu_{n, p}+a \sigma_{n, p} \\
& \sigma_{n, p, 0}=b \sigma_{n, p}
\end{aligned}
$$

olarak tanımlanmıştır.

Deo yaptığı çalışma ile $\boldsymbol{\sigma}_{0}^{2}$ sonuçları ile Eşitlik (10)‘daki aynı sınır dağılımı içinde yer değiştirerek geliştirebileceğini göstermiştir (Deo, 2016; Ciflikli \& Ilgin, 2017). Problemi daha iyi anlamak için standart 1. dereceden Tracy-Widom değişkeninin ele alınması uygundur,

$$
E\left(\hat{\lambda}_{1}^{s}\right)=E\left[\frac{\hat{\lambda}_{1}^{s}}{\sigma_{0}^{2 s}}\right]\left(\sigma_{0}^{2 s}\right)=E\left(\frac{\hat{\lambda}_{1}^{s}}{\sigma_{0}^{2 s}}\right) E\left(\sigma_{0}^{2 s}\right)
$$

Burada $\hat{\lambda}_{1}$ geliştirilmiş en büyük özdeğerin standart versiyonudur. Hesaplamalarda kullanılan, $\lambda_{1} / \sigma_{0}^{2}$ 'nin $\boldsymbol{\sigma}_{0}^{2}$ 'den bağımsız olması gerektiğinden herhangi pozitif tamsayı $s$ için,

$$
E\left(\frac{\hat{I}_{1}^{s}}{\widehat{\sigma}_{0}^{2 S}}\right)=\frac{E\left(\hat{I}_{1}^{s}\right)}{E\left(\widehat{\sigma}_{0}^{2 S}\right)}
$$

eşitliği yazılabilir. $\widehat{\boldsymbol{\sigma}}_{\mathbf{0}}^{2}$ 'nin $\sigma^{2} X_{n p}^{2} /(\mathrm{np})$ 'den bağımsız dağıtılmış olmasından dolayı $E\left(\sigma_{0}^{2}\right)=\sigma^{2} \quad$ ve $E\left(\hat{\sigma}_{0}^{4}\right)=2 \sigma^{4} / n p$ olmaktadır. $\hat{\lambda}_{1}^{\prime}$ 'in tam olarak ortalaması ve varyansını bilinmediğinden asimtotik yaklaşım kullanılarak $E=\grave{\lambda}_{1} / \sigma^{2}$ ve $E=\left(\lambda_{1}^{2} / \sigma^{4}\right)=$ $\sigma_{n, p, 0}^{2}+\mu_{n, p, 0}^{2}$ olduğundan bu değerler Eşitlik (11)'de yerine yazılırsa son olarak düzeltilmiş varyans $\sigma_{c}^{2}$ elde edilir.

$$
\sigma_{c}^{2}=\operatorname{var}\left(\frac{\lambda_{1}}{\sigma_{0}^{2}}\right)=\frac{n p}{2+n p}\left(\sigma_{n, p, 0}^{2}-\mu_{n, p, 0}^{2}\right)
$$

ve bu durumda düzeltilmiş varyansla birlikte $L_{C}$ istatistiği aşağıdaki gibi olur.

$$
L_{C} \equiv\left(\dot{\lambda}_{1} / \sigma^{2}-\mu_{n, p}\right) / \sigma_{n, p, C} \stackrel{D}{\rightarrow} T W_{1}
$$

Böylece Eşitlik (13) ile verilen özdeğer dağılımı, Wishart matrisleri için yapılmış en güncel çalışma olmasıyla beraber benzetim çalışmaları ile de $\mathrm{TW}_{1}$ dağılımına en fazla benzeyen dağılımdır.

\section{Araştırma Sonuçları ve Tartışma}

\subsection{Bulgular}

Yapılan benzetim çalışmalarında Rassal olarak oluşturulan 0 ortalamalı matrisler için kovaryans matrisleri hesaplanmış ve bu matrislerin en büyük özdeğer dağılımları için 2. Bölümde verilen fonksiyonlar temel alınmıştır. Ayrıca yapılan benzetim çalışmalarında Monte Carlo analizi için yöntemler 1000 kere çalıştırılmıştır. Sonuçların daha iyi yorumlanması için Ma, Deo, Jhonstone tarafından yapılan çalışmalar ile $\mathrm{TW}_{1}$ olasılık dağılımı aynı şekillerde verilmiştir.

Şekil 1'de $n=500, p=2$ için $L_{j}, L_{m a}, L_{\text {deo }}$ istatistikleri verilmektedir. Görüldüğü üzere $\mathrm{TW}_{1}$ olasılık dağılımının merkezi yaklaşık olarak x ekseninde -1.38 'de bulunmaktadır. Fakat benzetim çalışmalarında kullanılan diğer dağılım fonksiyonları Ma için -9.5, Jhonstone için -9.6 fakat Deo için yaklaşık -7 seviyesindedir. Deo olasılık dağılımı TW $\mathrm{TW}_{1}$ dağılımına çok yakınsamamakla birlikte 
diğerlerinden daha doğru sonuç verdiği görülmektedir. Deo'nun önerdiği dağılımın yakınsama başarısı Eşitlik 1'e göre $n$ ve $p$ değerlerine doğrudan bağlıdır.

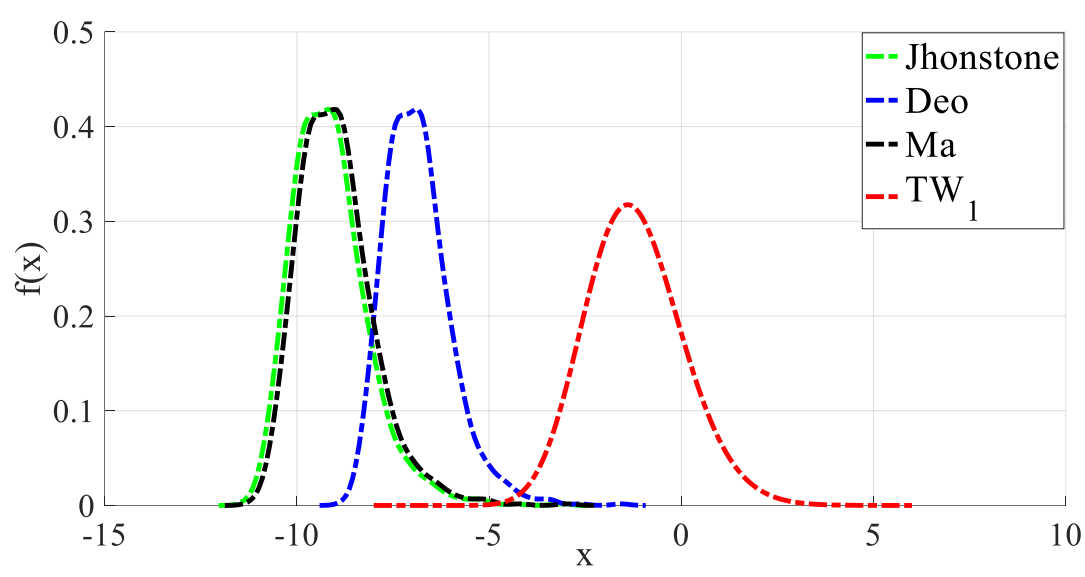

Şekil 1. $n=500, p=2$ için $L_{j}, L_{d e o}, L_{m a}$ Istatistikleri

$n=1000, p=4$ değerlerinin benzetim sonuçları için Şekil 2'ye bakılabilir. Burada göze çarpan en büyük değişiklik, verilen bütün dağılımların Şekil 1 ile kıyaslandığında $\mathrm{TW}_{1}$ 'e daha fazla yakınsadığıdır.

Özellikle Deo tarafından öne sürülen dağılımın diğerlerine göre oldukça başarılı olduğu görülmektedir. Grafiğe dikkat edilirse dağılımların varyansı hemen hemen birbirlerine benzemekle beraber, gerçek farkın dağılımların ortalamasında olduğu görülmektedir.

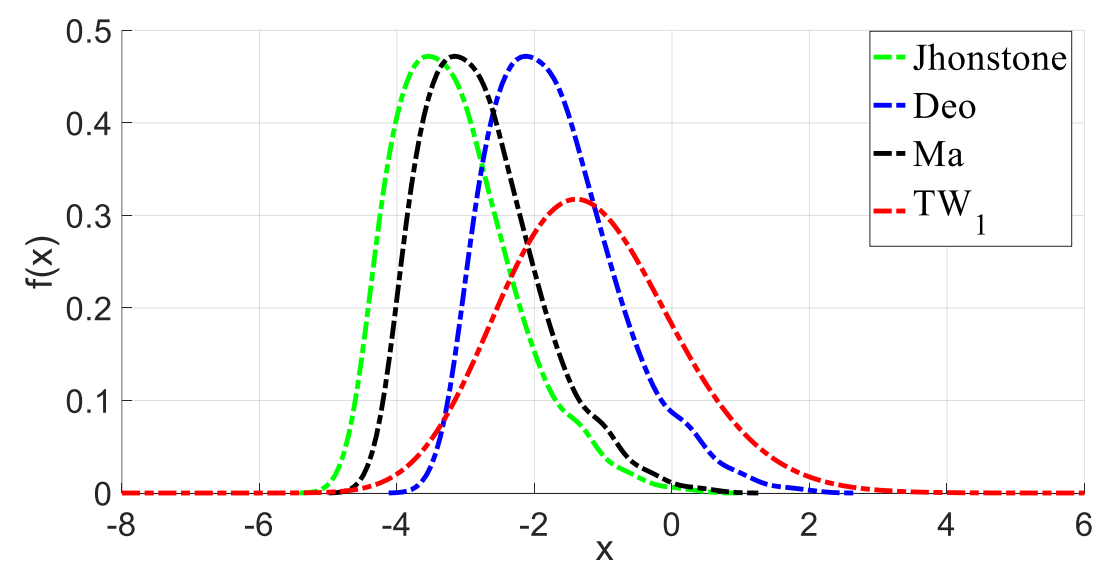

Şekil 2. $n=1000, p=4$ için $L_{j}, L_{m a}, L_{d e o} \dot{I}$ statistikleri

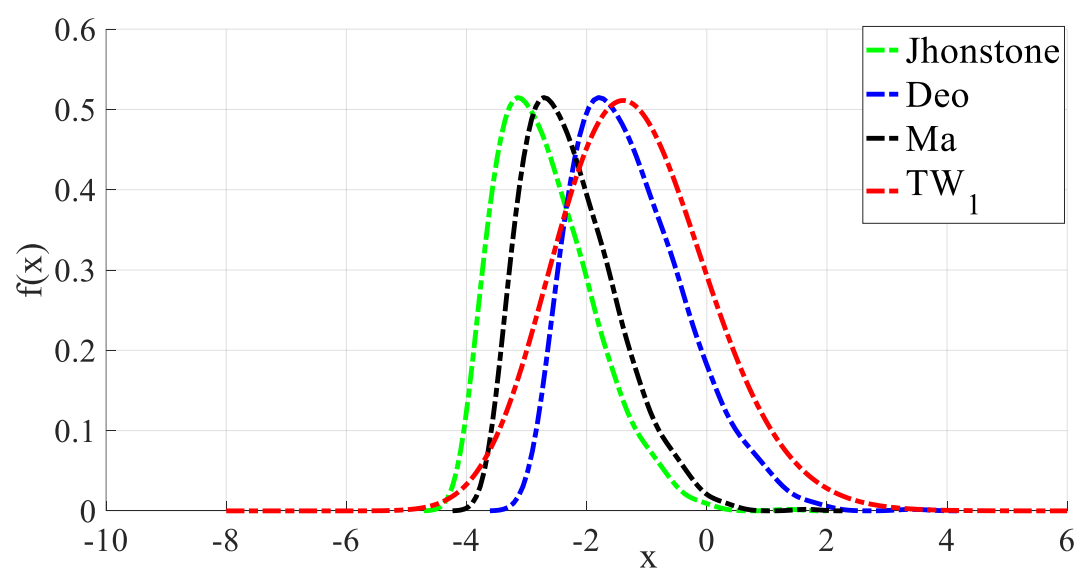

Şekil 3. $n=10000, p=8$ için $L_{j}, L_{m a}, L_{d e o}$ Istatistikleri 
Şekil 3’te ise bu çalışmada kullanılan en büyük Wishart matrisi için benzetim sonuçları verilmektedir. Deo tarafindan yapılan çalışmanın, TW' 'e oldukça benzediği görülmektedir.

Verilen şekillerdeki dağılımların ortalama değerlerinin 1. derece Tracy-Widom dağılımından ne kadar saptı̆̆ını görmek amacıyla

$$
\begin{aligned}
& \mu_{(L j)}=\left|\mu_{T W 1}-\mu_{L j}\right| \\
& \mu_{(L c)}=\left|\mu_{T W 1}-\mu_{L c}\right|
\end{aligned}
$$

eşitlikleri kullanılmıştır. Tablo 1'de standart 1. dereceden Tracy-Widom dağılımının merkezi ile Ma, Deo ve Jhonstone tarafından önerilen yöntemlerin karşılaştırılması tabloda gösterilmektedir. Tabloya bakıldığında Deo tarafindan önerilen dağılım fonksiyonunun bütün matrisler için diğerlerinden daha doğru sonuçlar verdiği görülmektedir. Örneğin 4x1000 boyutunda bir matris için, $\mu_{(M a)}$ değerinin 1.72 olduğu görülmektedir. Aynı ölçütteki matris için $\mu_{(L j)}$ değeri 2.1 olarak ölçülmüştür.

Tablo 1. Dağllımların Ortalama Değerleri

\begin{tabular}{|c|c|c|c|}
\hline Boyut & $\mu_{(L j)}$ & $\mu_{(M a)}$ & $\mu_{\left(D e o=L_{C}\right)}$ \\
\hline $2 \times 500$ & 7.75 & 7.72 & 5.62 \\
\hline $2 \times 1000$ & 7.37 & 7.35 & 5.17 \\
\hline $2 \times 10000$ & 6.51 & 6.45 & 4.14 \\
\hline $4 \times 500$ & 2.29 & 1.83 & 0.79 \\
\hline $4 \times 1000$ & 2.1 & 1.72 & 0.72 \\
\hline $4 \times 10000$ & 1.84 & 1.45 & 0.57 \\
\hline $8 \times 500$ & 2.18 & 1.67 & 0.83 \\
\hline $8 \times 1000$ & 2.01 & 1.54 & 0.69 \\
\hline $8 \times 10000$ & 1.77 & 1.27 & 0.44 \\
\hline
\end{tabular}

\section{Sonuç}

$\mathrm{Bu}$ çalışmada yapılan benzetim çalışmaları sonucunda literatürde bulunan bazı özdeğer dağılım fonksiyonlarının benzetim sonuçları verilmektedir. Böylece Bilişsel Radyo veya radar sistemleri için farklı uygulamalarda kullanılan özdeğer dağılım fonksiyonlarının başarımları incelenmiştir. Özdeğer dağılım fonksiyonları özellikle Bilişsel Radyo uygulamalarında spektrum algılama yöntemleri için oldukça önemlidir. Bu uygulamalarda standart özdeğer oranı gibi test istatistikleri sıklıkla kullanılmaktadır. $\mathrm{Bu}$ nedenle belirli matrislerin özdeğer dağılımlarını kapalı fonksiyonlarla ifade etmek için literatürde oldukça fazla çalışma yapılmıştır. Bu çalışma temelinde özdeğer dağılımı için verilen bazı kapalı fonksiyonların başarımlarının karşılaştırılması temel alınmıştır. Yapılan benzetim çalışmaları Jhonstone, Ma ve Deo tarafından önerilen özdeğer dağılımını temel almaktadır. Yapılan benzetim çalışmaları sayesinde Deo tarafindan önerilen en büyük özdeğer dağılım fonksiyonu, Wishart matrislerinin en büyük özdeğer dağılımını $\mathrm{TW}_{1}$ ’e en fazla yakınsayan fonksiyon olduğunu kanıtlanmıştır.

\section{Kaynaklar}

Chen, Z., \& Zhang, Y. (2018). Cooperative energy detection algorithm based on background noise and direction finding error. AEUInternational Journal of Electronics and Communications, 326-341.

Ciflikli, C., \& Ilgin, F. Y. (2017). Bilişsel radyo sistemleri için kör spektrum algılama yöntemlerinin farklı haberleşme kanallarındaki performans analizi. Uluslararası Multidisipliner Çalışmalar ve Yenilikçi Teknolojiler Sempozyumu, (s. 251-252). Tokat. 
Ciflikli, C., \& Ilgin, F. Y. (2017). Bilişsel radyo sistemleri için özdeğer tabanlı algılama yöntemlerinde performans analizi. Bilge International Journal of Science and Technology Research, 32-37.

Ciflikli, C., \& Ilgin, F. Y. (2017). On the performance analysis of blind spectrum sensing methods for different communication channels. International Research Journal of Engineering and Technology, 1-5.

Ciflikli, C., \& Ilgin, F. Y. (2018). Covariance based spectrum sensing with studentized extreme eigenvalue. Tehnički vjesnik, 100-106.

Ciflikli, C., \& Ilgin, F. Y. (2020). Multiple antenna spectrum sensing based on glr detector in cognitive radios. Wireless Personal Communications, 1915-1927.

Deo, R. S. (2016). On the Tracy-Widom approximation of studentized extreme eigenvalues of Wishart matrices. Journal of Multivariate Analysis, 265-272.

Edelman, A. (2005). Random matrix theory. Acta Numer, 1-65.

Johansson, K. (2007). From gumbel to Tracy-Widom. Probability Theory and Related Fields volume, 75-112.

Johnstone, I. M. (2001). On the distribution of the largest eigenvalue in principal components analysis. Annals of statistics, 295-327.

Ma, Z. (2012). Accuracy of the Tracy-Widom limits for the extreme eigenvalues in white Wishart matrices. Bernoulli, 322-359.

Mohammadi, A., Javadi, S. H., Ciuonzo, D., Persico, V., \& Pescapè, A. (351). Distributed detection with fuzzy censoring sensors in the presence of noise uncertainty. Neurocomputing, 196-204.

Nadler, B. (2011). On the distribution of the ratio of the largest eigenvalue to the trace of a Wishart matrix. Journal of Multivariate Analysis, 363-371. 